\title{
167. Chirurgische Therapie der chronischen Pankreatitis: Zunehmende Tendenz zum nichtresezierenden Vorgehen
}

\author{
K. D. Rumpf, R. Pichlmayr und H. J. Mitzkat \\ Klinik für Abdominal- und Transplantationschirurgie und Abteilung Diabetologie, Medizinische Hochschule \\ Hannover, Konstanty-Gutschow-Straße 8, D-3000 Hannover 61
}

\section{Surgical Therapy of Chronic Pancreatitis: Tendency Toward Nonresecting Procedures}

\begin{abstract}
Summary. With regard to the most suitable operative procedure, 87 cases of partial duodenopancreatectomy versus 28 cases of nonresecting operations on the pancreas were compared and discussed. The nonresecting procedure consists of a longitudinal pancreaticojejunostomy combined with a technique we developed ourselves: the transduodenal pancreaticoplasty. Operative time and complications were favorable in this procedure and lethality and long-term results similar. The levels of serum insulin in provocation tests were shown to be increased immediately after the operation and at a 2-year follow-up. Pancreatic duct-system decompression shows promise of being a preferable alternative to pancreatic head resection.
\end{abstract}

Key words: Chronic pancreatitis - Pancreas/surgical therapy - Diabetes mellitus.

Zusammenfassung. Zur Frage des geeigneten Operierens bei chronischer Pankreatitis wurden 87 partielle Duodenopancreatectomien mit 28 nichtresezierenden Operationen verglichen. Letztere sind als longitudinale Pancreaticojejunostomie in Kombination mit einer eigenen Technik, der transduodenalen Pancreaticoplastik, operiert worden. Operations-Dauer und Komplikationsraten liegen beim nichtresezierenden Vorgehen günstiger, Letalität und Spätergebnisse sind gleich. Die Insulinmessung im Provokationstest zeigt eine Abnahme durch die P-Kopfresektion, im anderen Fall einen Insulinanstieg. Diese Tendenz wurde über 2 Jahre beobachtet. Insgesamt spricht vieles für ein konsequent sekretableitendes Operieren als Alternative zur Whippleschen Operation.

Schliisselwörter: Chronische Pankreatitis - Pankreas/chirurgische Therapie - Diabetes mellitus.

168. G. Thoma, V. Schumpelick, K. Hrynyschyn, Hamburg: Klinische Erfahrungen mit der biliodigestiven Anastomose nach Rodney-Smith.

Manuskript nicht eingegangen

\section{Die adjuvante Chemotherapie der Echinokokkose}

\author{
R. Bähr, G. Gaebel und H. Bockhorn \\ Chirurgische Universitätsklinik Tübingen, Calwer Straße 7, D-7400 Tübingen
}

\section{Adjuvant Chemotherapy of Echinococcosis}

Summary. Operative treatment of human echinococcosis is still the therapy of choice. Nevertheless, important progress has been made using chemotherapy with Vermox. Chemotherapy is reserved for patients with local inoperability, patients who cannot undergo a radical operation, especially in cases of Echinococcus multilocularis of the liver. Results and experience of drug therapy of Echinococcus multilocularis are presented.

Key words: Echinococcosis - Drug therapy with mebendazol.

Zusammenfassung. Die operative Behandlung der menschlichen Echinokokkose stellt nach wie vor die Therapie der Wahl dar. Dennoch konnten durch die Chemotherapie mit Vermox erhebliche Fortschritte erzielt werden. Die Chemotherapie ist für Patienten reserviert, bei denen technische Inoperabilität vorliegt, die nicht radikal operiert werden können bzw. die Radikalität des Eingriffes fraglich erscheint, vor allem beim Echinococcus multilocularis der Leber. Ergebnisse und Erfahrungen der medikamentösen Behandlung bei der Behandlung des Echinococcus multilocularis und granulosus werden mitgeteilt.

Schlüsselwörter: Echinokokkose - Medikamentöse Therapie mit Mebendazol. 